\author{
Karthik K. Bodla \\ Justin A. Weibel \\ Suresh V. Garimella ${ }^{1}$ \\ e-mail: sureshg@purdue.edu \\ Cooling Technologies Research Center, \\ NSF IUCRC, \\ School of Mechanical Engineering \\ and Birck Nanotechnology Center, \\ Purdue University, \\ West Lafayette, IN 47907-2088
}

\section{Advances in Fluid and Thermal Transport Property Analysis and Design of Sintered Porous Wick Microstructures}

\begin{abstract}
Sintered porous structures are ubiquitous as heat transport media for thermal management and other applications. In particular, low-porosity sintered packed beds are used as capillary-wicking and evaporation-enhancement structures in heat pipes. Accurate prediction and analysis of their transport characteristics for different microstructure geometries is important for improved design. Owing to the random nature and geometric complexity of these materials, development of predictive methods has been the subject of extensive prior research. The present work summarizes and builds upon past studies and recent advances in pore-scale modeling of fluid and thermal transport within such heterogeneous media. A brief review of various analytical and numerical models for simplified prediction of transport characteristics such as effective thermal conductivity, permeability, and interfacial heat transfer is presented. More recently, there has been a growing interest in direct numerical simulation of transport in realistic representations of the porous medium geometry; for example, by employing nondestructive $3 D$ imaging techniques such as X-ray microtomography. Future research directions are identified, looking beyond techniques intended for material characterization alone, and focusing on those targeting the reverse engineering of wick structures via modeling of the physical sintering fabrication processes. This approach may eventually be employed to design intricate sintered porous structures with desired properties tailored to specific applications. [DOI: $10.1115 / 1.4023569]$
\end{abstract}

Keywords: porous media, sintered materials, direct simulation, microtomography, reverse engineering, electronics cooling, thermal and fluid transport

\section{Introduction}

Naturally occurring and engineered porous media find use in a wide range of construction materials, prosthetics, energy storage, and thermal management applications. Analysis of transport, viz. fluid flow, heat transfer, species transport, and other mechanisms, in porous media is thus critical for many industries. Owing to their generality and wide applicability in engineering [1], single-phase and two-phase fluid and thermal transport through porous media have been commonly studied.

One application in which porous media have found ubiquitous use is in the thermal management of electronic devices. Today's high heat-flux dissipating and densely packed electronic equipment, requiring dissipation of as high as $500 \mathrm{~W} / \mathrm{cm}^{2}$ [2] in military motion-tracking systems and automotive power electronics, pose an increasing need to design optimized and efficient cooling solutions [3]. In these situations, liquid-vapor phase-change heat transfer is an attractive cooling mechanism owing to its near isothermal operation, and is therefore employed in a number of jet impingement, microchannel, and immersion cooling solutions $[4,5]$. Porous media are also commonly used as both evaporation/ boiling and convective heat transfer enhancement surfaces.

Several different augmentation techniques have been proposed in the literature to enhance active and passive heat transfer mechanisms, and the interested reader is referred to comprehensive reviews on the subject $[6,7]$. Because of characteristics such as

\footnotetext{
${ }^{1}$ Corresponding author.

Manuscript received December 7, 2012; final manuscript received January 23 , 2013; published online May 16, 2013. Assoc. Editor: Leslie Phinney.
}

intricate three-dimensional structure, large surface area to volume ratio, continuously changing flow area, and stochastic, tortuous flow paths, porous media can greatly enhance convective heat transfer $[1,8]$ and evaporation/boiling processes [2,9]. With advances in manufacturing and processing technologies for fabricating porous materials, there has been a recent surge in the number of studies on characterization of heat transfer and fluid flow through porous media. In this work, we focus on low-porosity sintered wicks as used in thermal management applications.

Sintered wick structures demonstrate several desirable characteristics. In addition to having a large surface area to volume ratio, they also possess the ability to passively transport fluids via capillary action under adverse gravity conditions, thereby finding use in capillary pumped loop systems (CPLs) and heat pipes $[9,10]$. Wicks are employed with a variety of morphologies and materials. Simple groove structures are common in heat pipes, but stochastic porous sintered materials composed of felts, screens, and powders often outperform their counterparts $[10,11]$. Of these, sintered copper powder wicks are widely used due to their low manufacturing cost, high effective thermal conductivity, and superior capillary pressure to permeability ratio [11]. Additionally, the highly tortuous interstitial pore paths allow capillary supply under boiling conditions and are ideal for use in high heat flux-dissipating vapor chamber heat spreaders. Recently, Weibel et al. [2] experimentally investigated evaporation and boiling from the surface of porous sintered powder wick structures and demonstrated dissipation in excess of $500 \mathrm{~W} / \mathrm{cm}^{2}$ before dryout. Various microstructure configurations for the sintered copper powder have also been explored in the literature. Biporous [12,13] and micro-patterned [14] sintered powder wicks were successfully shown to exhibit superior performance owing to 
the presence of two size scales-larger pores provided a less restrictive vapor escape path, while the smaller pores improved the capillary resupply of liquid to nucleation sites.

Extensive reviews of the analysis of fluid flow and thermal transport through porous media are available $[1,15,16]$. The past reviews are not revisited here; rather, we focus on recent advances, specifically with regard to accurate prediction and modeling of transport through porous sintered media that are relevant to thermal management applications. In the following sections, various analytical/semiempirical, semirealistic unit-cell, and realistic porous media representations and transport analysis methodologies are discussed in detail. A brief outlook is provided on the future directions in tailored reverse engineering of such porous media for optimized, application-specific performance.

\section{Analytical and Semiempirical Modeling}

Extreme randomness largely restricts the study of porous media to experimental methods. From a modeling perspective, much of the extant work was restricted to simplified analytical or semiempirical approaches [17,18]. In these approaches, the exact pore-scale transport mechanisms and experimentally observed performance are averaged over a suitable representative elementary volume (REV) for which macroscale governing equations can be formulated [8]. The REV consists of a collection of particles and pores, and the porous medium is represented as a collection of many such REVs. The REV scale is selected so that it is large enough to be representative of the bulk properties, while at the same time being significantly smaller than the device size [8]. Figure 1 shows a typical REV, along with the various length scales for a vapor chamber heat spreader application. Using this approach, entire device-level analysis may be performed on a simplified volume-averaging basis without the need to resolve transport in geometries down to individual pores/particles. This averaging procedure leads to a closure problem, however, wherein dispersion, tortuosity, and interfacial transport quantities need to be suitably modeled. Several closure relations have been proposed in the literature for various types of porous media that contain different geometry-specific constants/coefficients. This section focuses on simplified analytical and empirical prediction of these REV quantities for sintered media. Furthermore, a brief overview of fractal analysis models, recently developed for predicting transport characteristics of porous media, is also provided.

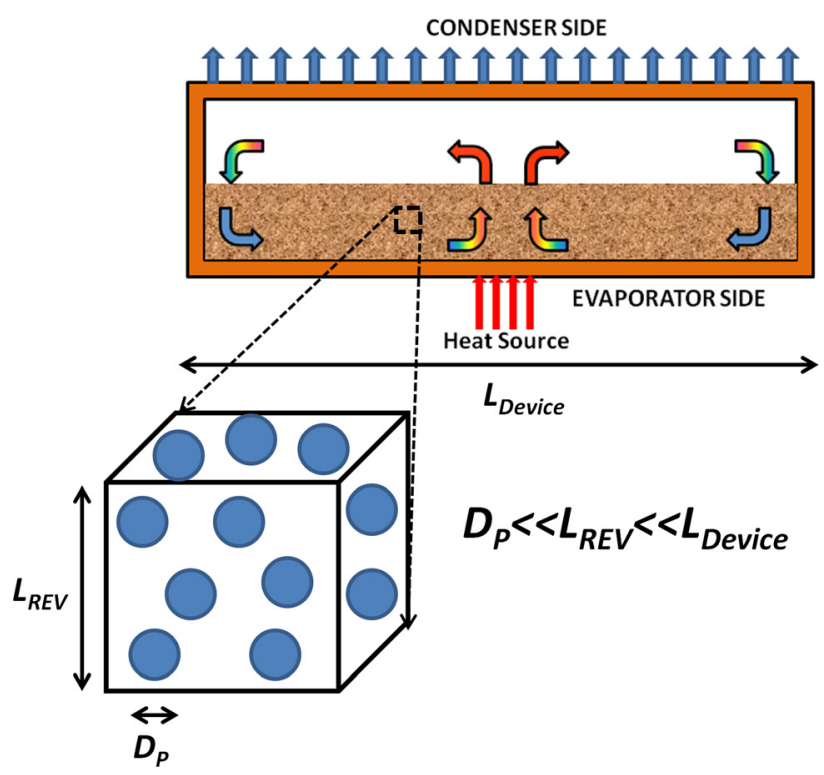

Fig. 1 Various device, representative elementary volume, and pore length scales shown for a typical vapor chamber heat spreader application
It may be noted that this REV approach is not exclusive to empirical prediction of porous medium transport properties. For ease of implementation and computational efficiency, most numerical approaches such as direct simulation of pore-scale transport eventually translate outputs into an REV-based implementation at the device scale. By employing such an REV-based approach, devicelevel performance may be readily analyzed. For example, the performance of energy storage systems [19] has been modeled in this manner. Furthermore, nonporous geometries may also be easily included and coupled to porous structures without the need to model the pore-scale geometry. In the following sections, prediction of single-phase fluid flow and heat transfer through porous sintered beds is discussed, along with a brief consideration of models for two-phase characteristics of interest, viz., capillary pressure and evaporative heat transfer.

2.1 Effective Thermal Conductivity. Effective thermal conductivity of a porous material is an important thermal property for applications such as heat pipes and heat sinks. Many of the recommended effective thermal conductivity models for sintered beds have been based on analytical expressions derived for simplified representations of packed bed geometries [10]. The widely used Maxwell-Eucken and effective medium theory (EMT) models for thermal conductivity are discussed in Carson et al. [20], and with respect to sintered beds, in Bodla et al. [21]. These models have a single free parameter - the porosity of the porous material — and are correlated with respect to thermal conductivity of the solid and pore phases. Bodla et al. observed that, when used for sintered powders, the EMT model performed more favorably than the MaxwellEucken models ( $\sim \pm 50 \%$ deviation observed for the EMT model as against $\pm 200 \%$ for the Maxwell-Eucken models [21]).

Kaviany [1] provided a detailed description of several effective thermal conductivity models for spherical particle beds, and compared their predictions against the experimental results of Nozad et al. [22] and Prasad et al. [23]. Alexander [24] provided the following empirical correlation for predicting effective thermal conductivity of sintered beds as a function of porosity:

$$
k_{\text {eff }}=k_{f}\left(\frac{k_{s}}{k_{f}}\right)^{\varepsilon^{\delta}}
$$

Here, $\delta$ is an empirical fitting parameter with a value of 0.53 for sintered powders. Furthermore, Atabaki and Baliga [25] modified a correlation originally developed for cold-pressed powdered metals [26] to include the effects of porosity and solid-to-fluid thermal conductivity ratio. Their correlation performs reasonably well when compared against experimental data on nickel and stainless steel sintered powders [25], and sintered copper powder [27].

2.2 Permeability and Interfacial Heat Transfer. Permeability of a porous material is measured by correlating the observed pressure drop to the superficial velocity of the fluid, and employing modifications to Darcy's law [1]. A popular form of this relationship is the Forchheimer equation, defined as:

$$
\left(-\frac{\partial P}{\partial x}\right)=\frac{\mu}{K} U+\frac{\rho C_{E}}{\sqrt{K}} U^{2}
$$

The first term on the right hand side represents the viscous component of pressure drop, while the second term represents the inertial component. For hydrodynamic flow resistance prediction, some of the widely used models for sintered beds are the Carman-Kozeny correlation $[1,10]$ and the modified Ergun's equation proposed by Macdonald et al. [28]. The performance of these models in predicting pressure drop through porous sintered wicks is discussed in detail in Bodla et al. [21]. These models were found to underpredict pressure drop (and correspondingly overpredict permeability) when used for analyzing transport through sintered media, as 
they were originally developed for simple packed beds of spherical particles. Further discussion on these discrepancies is provided in Sec. 4.2.3

Wakao and Kaguei [29] compiled and compared results from various transient and steady-state experiments pertaining to interstitial heat transfer in spherical packed beds, and proposed a correlation to predict Nusselt number as a function of Reynolds and Prandtl numbers. Their model contains an additive constant that represents the conduction limit of the Nusselt number usually observed in densely-packed porous media. Suitable modifications to this model to account for interparticle contact in the sintered beds were proposed by Bodla et al. [21].

2.3 Capillary Pressure and Evaporative Heat Transfer. Apart from permeability and thermal conductivity, additional characteristics of wick microstructures such as capillary pressure and evaporative performance also require characterization for heat pipe applications. For macroscopic device-level models, studies often rely on experimental measurements, and no simple correlations relating evaporation characteristics to wick microstructure exist. Iverson et al. [30] experimentally quantified evaporation rates from sintered wick samples under conditions simulating heat pipe operation. Furthermore, Davis and Garimella [31] measured evaporative thermal resistance of wicks of different porosities under saturated vapor conditions, while Migliaccio and Garimella [32] mapped out the evaporative performance of an organized bed of copper spheres. Hanlon and Ma [33] performed experiments and developed a simple numerical model to delineate the role of thin-film evaporation in sintered media.

Capillary pressure may be measured with various experimental techniques, which focus on determining an effective pore radius of the structure. This information can then be used to calculate capillary pressure for any liquid given the properties and wetting characteristics, using the modified Young-Laplace equation:

$$
\Delta P_{\text {cap }}=\frac{2 \sigma}{r_{\text {eff }}} ; \quad r_{\text {eff }}=\frac{r_{p}}{\cos (\theta)}
$$

One such commonly used technique, known as the bubble-point method provides a conservative estimate of capillary pressure $[10,34]$. On the other hand, the maximum capillary pressure provided by the wick structure may be determined by measuring the gravitational head needed to break the liquid column in a plug of wick material saturated with liquid $[35,36]$. Another technique, known as the rate of rise test, observes the transient rise of liquid in a sample for estimating the effective pore radius [34,37].

2.4 Fractal Analysis of Transport. Fractal analysis provides an alternative and useful means of analyzing flow and transport properties of porous materials. Katz and Thompson [38] provided experimental evidence indicating that the pore space in porous sandstone has a fractal nature, and is self-similar over three to four orders of magnitude ranging from $10 \AA$ to $100 \mu \mathrm{m}$. A number of studies have employed fractal theory to analyze transport through porous media. Such analyses have been performed for a variety of porous materials ranging from soil (e.g., Tyler and Wheatcraft [39]) to biological porous materials such as tree networks, stems, leaves and bronchial networks (e.g., Majumdar [40] and Kitaoka et al. [41]). Only a brief introduction to fractal analysis is provided here, and the interested reader is referred to comprehensive reviews on the subject (e.g., Yu [42]) for a detailed discussion.

Porous media such as packed beds, sandstone, and heat pipe wicks have been shown to be fractal in nature, consisting of numerous irregular pores of different sizes spanning several orders of magnitudes in length scale [42], and fractal models have been successfully proposed for estimating fundamental transport characteristics such as tortuosity, effective thermal conductivity and permeability. Ma et al. [43] proposed a novel self-similarity model for predicting effective thermal conductivity of porous media. In this model derived from a thermal-electrical conduction analogy, the porous medium is assumed to be statistically selfsimilar, with the defining parameters being porosity, thermal conductivity ratio, and contact resistance. The model was shown to predict effective thermal conductivity of a wide variety of porous materials accurately, across a range of solid-to-fluid thermal conductivity ratios from 0.1 to $10^{4}$ [43]. The model was later extended to three-phase porous media and was shown to be in close agreement with experimental measurements [44].

Flow characteristics of porous media have also been analyzed via fractal models. By assuming the porous medium to consist of a bundle of tortuous capillaries, Yu et al. $[45,46]$ derived fractal permeability models. The validity of this model was successfully tested for both particle-like and fibrous media [42]. Xu and $\mathrm{Yu}$ [47] derived an improved fractal permeability model, expressing permeability as a function of fractal dimensions, porosity and maximum capillary diameter. An analytical expression for the Karman-Cozeny constant was also derived. Their model accounts for microstructural information such as the fractal dimensions in addition to the porosity that is conventionally employed.

\section{Representative Unit Cell Modeling}

An alternative approach to obtaining the characteristics of porous media is to employ a microscopic, pore-scale model on a unit cell. Once estimated, these characteristics may be employed in a device-scale model for predicting performance. Such an approach has been employed in the literature $[48,49]$ for modeling heat pipes and vapor chambers. By feeding inputs such as effective thermal conductivity, permeability, capillary pressure, and evaporation characteristics from the microscale model, the performance of a novel, thin vapor chamber heat spreader was analyzed by Ranjan et al. [49]. This section discusses prediction of transport through approximated, unit-cell sized domains of porous materials.

Pore-scale models solve the governing equations within the liquid/solid domain at the pore/particle scale, and therefore require detailed microstructure geometry information. Most often, porous media exhibit random variability in geometric features such as particle size and shape in the case of sintered beds. However, at the device length scale, the large collection of such variable features exhibits a macroscopic periodic behavior under hydrodynamically and thermally fully developed conditions [18]. In such cases, periodic unit-cell modeling approximations may provide accurate performance prediction.

A number of approximate representations have been proposed and successfully employed to predict transport through random porous materials, based on the assumption of microstructural periodicity and a knowledge of the physical basis regarding the manufacturing process employed. These models consist of free parameters which are varied to closely match the characteristics of the porous media they represent. Being parameterizable, they may be readily reconstructed and employed for direct numerical simulation (DNS). The unit-cell modeling approaches that have been developed in the recent past to model conductive, convective and capillary transport, and thin-film evaporation characteristics of sintered beds and microcapillary pillar structures, are now discussed.

3.1 Conductive and Convective Transport. By solving for the potential field around a spherical particle, Maxwell [50] developed equations for predicting effective electrical conductivity of spherical packed beds, for the case of large particle separations, i.e., when the potential field near a particle is not influenced by the presence of a neighboring particle. Employing analogy between electrical and thermal conduction, similar relationships have been developed for effective thermal conductivity [20]. However, sintered particle beds consist of a neck region near the particle-particle contacts which reduces the overall thermal resistance. Models 
based on simple packing of spheres for representing sintered media neglect these necks, and hence, poorly predict effective thermal conductivity of realistic wick structures [21]. Instead, studies often rely on experimental/semiempirical correlations such as those presented earlier in Sec. 2.1, or on direct simulation of the exact geometry as will be discussed in Sec. 4. In a recent study by Dominguez Espinosa [51], the effect of the neck was included in BCC and FCC configurations of the spheres and the results were compared against experiments. The numerical model was observed to underpredict effective thermal conductivity, and differences in the range $15-26 \%$ were observed for the various samples.

For permeability and interfacial heat transfer, much work has been done for simple packed beds, and similar studies are often employed to model sintered beds. Comprehensive reviews on the subject are available (e.g., Refs. [1,8]). Kaviany [1] described several unit-cell models for estimating flow and heat transfer characteristics of simple close-packed beds. Ranjan et al. [52] numerically estimated the permeability of a square-packed arrangement of spheres by considering a single unit cell. Periodic boundary conditions were employed in the flow direction, while symmetry boundary conditions were imposed on boundaries transverse to the main flow direction. The computed permeability was found to be similar to the Carman-Kozeny correlation developed for spherical particle beds. Wicking and evaporation characteristics, as predicted by these unit cells models, are discussed below.

3.2 Wicking and Evaporation Characteristics. Commercial sintered powder wick structures have porosities in the range of 56-78\% [30]. Sintering usually begins with a close-packed powder compact. Monosized spheres arranged in a BCC, hexagonally close-packed (HCP), Weaire-Phelan (WP) or other close-packing configuration have a porosity of $\sim 26-36 \%$. Therefore, the significantly larger porosities exhibited by commercial samples are indicative of randomly shaped and sized particles. However, for the purpose of microscopic modeling, most studies are restricted to unit cells composed of single-sized spheres. Ranjan et al. [53] analyzed the wicking and thin-film evaporation characteristics of microstructures, such as square/hexagonally close-packed spheres and vertical/horizontal cylindrical wires. Variable porosities, solid-liquid contact angles, and liquid levels in the wick structure were analyzed for these microstructures. It was concluded that hexagonal packing of spheres, most representative of a sintered packed bed, provided the highest capillary pressure. Later, the study was extended to include several novel microstructures such as capillary porous media consisting of pyramidal and prismshaped pillars [52]. While hexagonally packed sphere structures generate the highest capillary pressure, it was observed that pyramidal pillars provide similar capillary pressure at significantly reduced pressure drops. Furthermore, the pyramidal structure produces an elongated thin-liquid film at the solid-liquid contact line. A kinetic theory-based [54,55] analysis of evaporation concluded that pyramidal micropillars perform the best when large liquid pressure drop is of concern. Figure 2 shows representative values of base heat transfer coefficient for square-packed unit cells consisting of conical, pyramidal, and cylindrical pillars as a function of contact angle. The pyramidal pillars demonstrate the highest evaporative heat transfer coefficient for both constant permeability and constant porosity cases, at any contact angle in the range 0-60 deg [52].

Furthermore, Ranjan et al. [53] also analyzed the effect of wick topology on the extent of thin-film area created at the solid-liquid contact line. Evaporation from the thin-film region of a liquid meniscus has been shown in a number of prior studies (e.g., Refs. $[32,56,57])$ to be the dominant mode of phase-change heat transfer from thin wick layers. Ranjan et al. [55] observed that an approximately $20 \%$ area of the meniscus near the triple line contributes to more than $80 \%$ of the overall evaporative heat transfer from the entire meniscus. Therefore, a simple geometric analysis of thinfilm area based on the static liquid meniscus shape provides

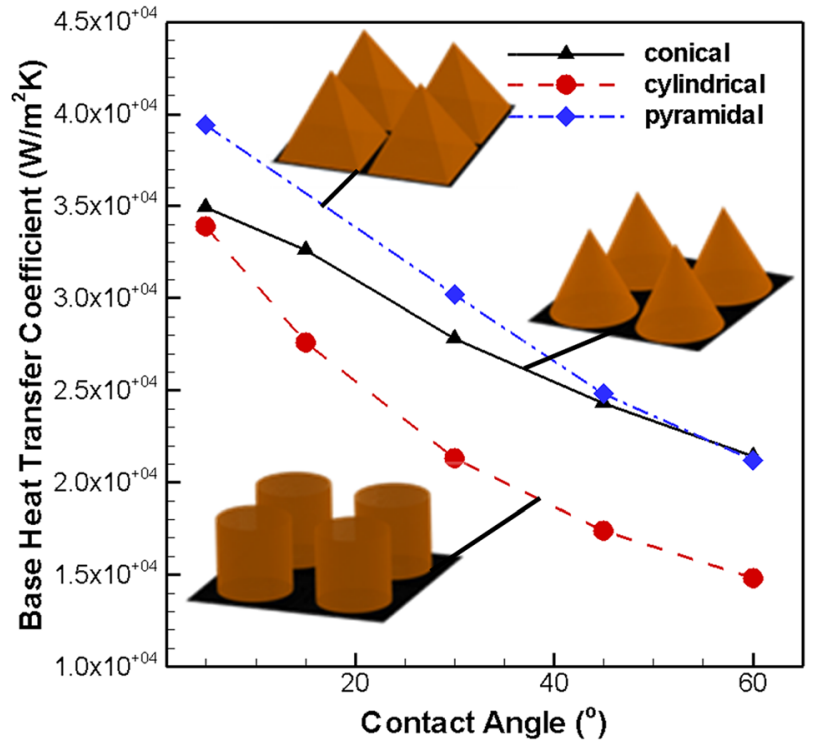

a)

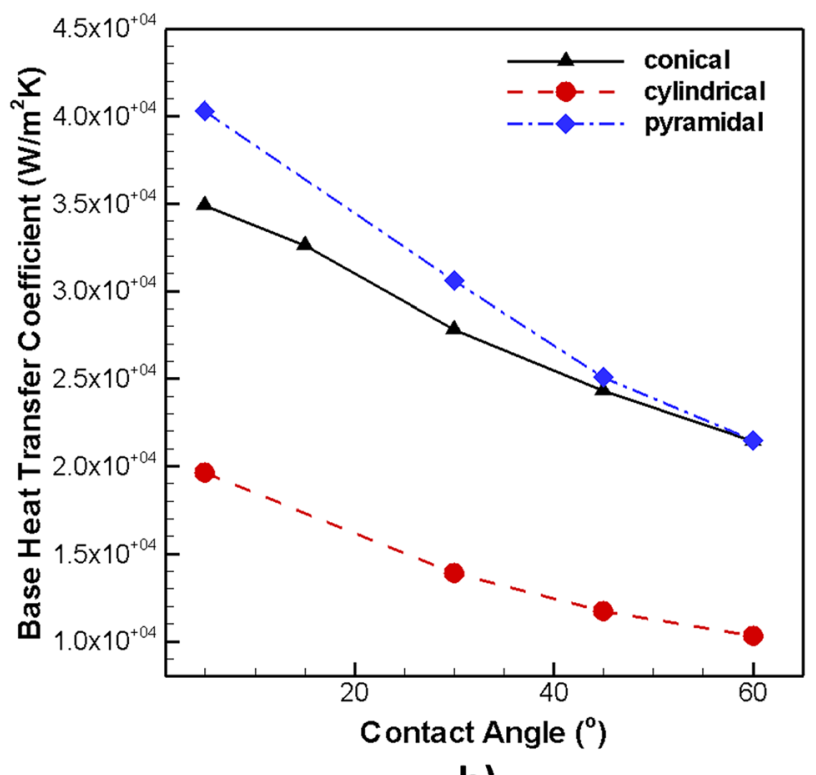

b)

Fig. 2 Evaporative base heat transfer coefficient as a function of contact angle for square-packed pyramidal, cylindrical,and conical pillars of radius and height equal to $100 \mu \mathrm{m}$, for (a) constant porosity, $\varepsilon=0.78$, and $(b)$ constant permeability,
$K=1.02 \times 10^{-9} \mathrm{~m}^{2}$

insight into evaporative performance without the need for a conjugate heat transfer analysis. It was concluded that the trends in thin-film area and capillary pressure generally correspond, and hexagonal close-packed spheres offer the most favorable geometry for evaporation.

\section{Direct Simulation of Realistic Microstructures}

The modeling approaches detailed above suffer from a number of inherent limitations. The semiempirical models rely on experimental measurements for determination of constants in the correlations. Similarly, unit-cell based porous media models are accurate only when the assumed geometry closely resembles the material it represents. The applicability of such models is limited for random and irregularly shaped porous media. For example, models representing sintered beds as closely packed spheres 
neglect the interparticle necking, and hence fail to capture the low thermal resistance of sintered beds compared to simple packed beds and cold-pressed beds [25]. Furthermore, commercially produced sintered media exhibit a distribution of pore/particle sizes; all unit-cell models previously discussed assumed a single characteristic size. To account for a realistic statistical variation, a collection of unit cells of varying dimensions must be considered.

An alternative modeling approach employs the actual random structure of the porous material as the computational domain for a pore-scale direct numerical simulation. Modeling the entire device in this manner is computationally impractical. It suffices instead to model a section consisting of a number of particles/pores that is representative of the bulk properties. The effective transport characteristics thus obtained may then be employed to assess the device-scale performance. Obtaining such complex, 3D geometrical information is not trivial, especially for materials wherein the actual physical features are on the scale of a few microns. In the following sections, microtomography, which has been widely employed for nondestructive 3D imaging of living biological samples, is described. In addition, recent developments concerning testing and 3D visualization of inorganic material, and its applicability in the present context, is discussed.

4.1 3D Reconstruction and Mesh Generation. Direct simulation of realistic 3D microstructures first requires imaging of the porous medium of interest using a suitable technique, such as microtomography. The resulting stack of images is processed to improve signal-to-noise ratio and to distinguish regions of the material and pore spaces. Subsequently, the segmented images are used to produce a computational mesh, representing the original scanned porous material [17]. The details of these steps are described in the following.

4.1.1 Microtomography-Based 3D Visualization. Extant twodimensional visualization and imaging techniques are at a mature state of development. Examples of such techniques include optical microscopy, scanning electron microscopy (SEM), and atomic force microscopy (AFM) [58]. These state-of-the-art techniques have high resolution and are widely used for material characterization, visualization and feature detection. Similar approaches for 3D analysis have, however, been limited, and 3D nondestructive visualization is an area of active research and development. Some of the earliest and commonly used 3D characterization methods employed destructive methods. In one such approach known as focused ion beam milling-based SEM (FIB SEM), the pore space in the medium to be imaged is impregnated with a solidifying resin. The resulting resin-saturated porous medium is milled and subsequently imaged in a series of steps [59]. The 3D structure of the porous material is visualized as a stack of aligned 2D sections. This approach is expensive, time consuming, and destructive. Moreover, depending on the pore and feature sizes, resin impregnation often requires high pumping pressures. Moreover, when the pores are closed, it is not possible to saturate the entire open volume, thereby limiting the utility of this approach.

An alternative, nondestructive approach traditionally used for analysis and visualization of biological samples is tomography [60]. Tomography refers to imaging by serial sectioning employing penetrating waves that interact with the object being imaged. Currently, there are a number of tomography methods based on X-rays, $\gamma$ rays, electrons, and ions [60], each with its merits and limitations. The methods based on X-rays and reconstruction algorithms involving mathematical transforms, known as computed tomography (CT), are the most widely used. Several variants of this method are available; the one that is useful in the present context is microcomputed tomography ( $\mu$-CT) or X-ray microtomography (XMT), which offers resolution on the order of a few microns [17].

XMT is based on the principle of differential absorptivities of materials to X-rays. The object to be scanned is irradiated with $\mathrm{X}$-rays and the resulting reflected beam is reconstructed using a set of algorithms such as fan or cone beam reconstruction [61].
The differential absorptivity (related to the material density) is reflected in the varying grayscale values of reconstructed images. Distinction based on a simple threshold can then be made to identify the different materials in the original object [17]. Further details on this technique may be found in Stauber and Muller [62]. This technique is steadily gaining popularity for accurate analysis and testing of intricate porous materials. In one of the earliest works employing XMT, Jasti et al. [63] analyzed Fountainebleau sandstones for the design of drilling fluids. This method has also been successfully employed in soil and petroleum research $[64,65]$. Apart from these, flow in realistic porous media may also be analyzed via techniques such as nuclear magnetic resonance (NMR). In one such study by Givler and Altobelli [66], the effective viscosity for the Brinkman-Forchcheimer flow model was determined for high-porosity foams. Furthermore, Gleeson et al. [67] employed NMR to image the pore space in limestone.

4.1.2 3D Mesh Generation From Images. Computational analysis of materials based on XMT data requires efficient and intelligent mesh generation algorithms for converting the scan (image) data into viable computational meshes. Various imagebased mesh generation methods have been proposed. Some of the earliest methods, popularly known as CAD-based approaches, were based on generation of a simple surface mesh from the scan data and subsequent meshing of the internal volume via conventional, commercial mesh generation packages [68]. These methods were found to be time-consuming, and virtually intractable for complex topologies such as random porous media. Voxel (volumetric pixel) element techniques (e.g., Ref. [69]) and marching cube-based meshing of scan data (e.g., Ref. [70]) provide more sophisticated alternatives. However, Watson et al. [71] reported these methods to be unreliable for complex geometries, although they worked relatively well for large volumes of materials with spherical pores. The studies discussed in the present work employ the meshing methodology proposed by Young et al. [68], which is implemented in the commercial image processing and mesh generation package, SIMPLEWARE [72]. This method produces robust, feature-preserving meshes even for complex geometries such as sintered porous beds. It may be noted that the image processing and mesh generation steps discussed here are presented in the context of the SIMPLEWARE package [72]. However, the procedure is expected to be similar for any equivalent approach.

The images obtained by scanning suffer from a number of artifacts and must first be processed before they can be used for mesh generation. Data processing steps include noise removal, region identification based on grayscale value, and 3D surface/volume reconstruction, prior to export in standard formats for CAD or mesh generation. The processing steps are only discussed briefly here and the readers are referred to the original references for details.

After noise removal, the solid and pore regions of interest are identified. The metal is much denser and has a higher X-ray absorptivity than the surrounding pore region, which is filled with air. This difference is reflected in the reconstructed image stack, where the brighter regions correspond to the metal and the darker regions to the surrounding pore. The solid-pore grayscale transition is blurred, and detection of discrete interfaces is defined based on a grayscale threshold value selected to ensure that the porosity of the reconstructed sample is the same as that of the original sample. The identified regions are then "stitched" with unit pixel separation among every two adjacent images in the 2D image stack to generate $3 \mathrm{D}$ volumes corresponding to the metal and pore domains of the original sintered copper sample. Unconnected regions and any existing islands are removed using the flood fill segmentation. The images are then down-sampled to reduce the number of elements in the final mesh. The typical workflow is summarized in Fig. 3 for the case of mesh generation from XMT data obtained for a sintered copper wick sample [21]. After processing the images, meshes may be generated using the proprietary image-based meshing algorithm in SIMPLEWARE [72]. Some of the distinguishing features of this algorithm are meshing of multiple domains with conformal mesh at the 


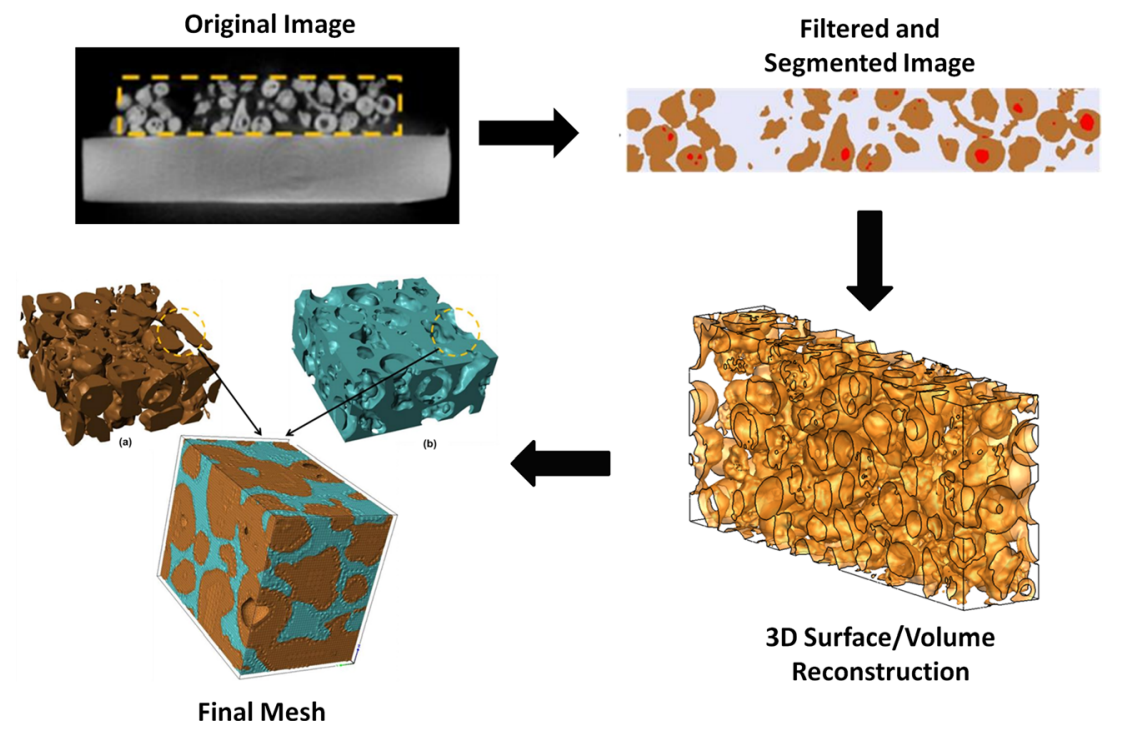

Fig. 3 Schematic illustration of various image processing and mesh generation steps, for typical image-based mesh generation. The demonstrated case corresponds to a sintered copper bed with particles of sizes $250-355 \mu \mathrm{m}$.

interface, ability to generate coarser elements in the interior relative to the interface and boundaries, and generation of all tetrahedral or mixed hexahedral and tetrahedral elements [68]. The following sections present results obtained with this approach for analysis of transport through porous sintered beds.

4.2 Conductive and Convective Transport. Three sintered copper powder wick samples employed in commercial heat pipes were characterized in Bodla et al. [21] in terms of their thermal transport properties-porosity, effective thermal conductivity, permeability, and interfacial heat transfer coefficient. These properties play an important role in evaluating capillary limit, a prevalent heat transfer limitation in heat pipes. Commercially produced samples of nearly identical porosities ( $\sim 61 \%$ open volume) were CT-scanned at $5.5 \mu \mathrm{m}$ resolution and the resulting image stack reconstructed to produce high-quality finite volume meshes for characterization.

4.2.1 Sample Preparation and Processing. Samples of approximate size $10 \mathrm{~mm} \times 5 \mathrm{~mm} \times 1-2 \mathrm{~mm}$ were cut from the original, commercial sintered copper powder samples using electric discharge machining (EDM) and imaged using a commercial axial $\mu$-CT scanner. This noncontact machining process was selected to ensure that the cuts did not introduce mechanical distortions in the sintered particles. The samples were made from different particle sizes $(45-75 \mu \mathrm{m}, 106-150 \mu \mathrm{m}$, and $250-355 \mu \mathrm{m})$ and had porosities of $61 \%, 64 \%$, and $63 \%$, respectively, as provided by the manufacturer (Thermacore, Inc.). The scanning resolution of $5.5 \mu \mathrm{m}$ was selected to resolve the individual pores and particles. The resulting stack of images was processed using the workflow described in Sec. 4.1.2. Some of the particles in the samples were found to be hollow; however, since the void fraction contributed by these cavities inside particles was less than $1 \%$ of the overall volume, this was included as part of the pore space. The number of particles/pores resolved in the scanned data was too large for meshing and subsequent CFD simulation. Consequently, the scanned domain was divided into 3-4 subvolumes, each with 15-35 particles along the longest dimension, and these subvolumes were meshed separately. This automatically accounted for some of the statistical variation exhibited by the samples.

The processed subvolumes were meshed with hexahedral elements using the robust "ScanFE-Grid" meshing algorithm in SIMPLEWARE [72], with volumetric and boundary mesh adaptation to reduce the mesh cell count without loss of fidelity. The smoothed conformal meshes thus generated are of very high qual- ity and consisted of approximately $20 \times 10^{6}$ cells for each subvolume (both metal and pore). A detailed discussion of the workflow and the parameters employed for image processing and meshing is available in Ref. [21]. The fluid and thermal transport characteristics of porous sintered beds obtained from the generated meshes are now presented.

4.2.2 Effective Thermal Conductivity and Necking Ratio. Effective thermal conductivity in direction $x_{i}$ is computed by imposing a temperature gradient across the ends of the sample while holding the lateral faces adiabatic. No pressure gradient is imposed, and the pores are filled with static fluid. The effective thermal conductivity is calculated as:

$$
k_{\mathrm{eff}}=\frac{-\int \boldsymbol{J} \cdot d \boldsymbol{A}}{\left(\frac{\partial T}{\partial x_{i}}\right) A}
$$

It is noted that the effective thermal conductivity is calculated by considering conduction through both the solid and fluid regions, i.e., the integral is performed on both the solid and fluid regions of the face, and $A$ is the total facial area. Furthermore, for particles found to be hollow, the closed void space is assumed to be filled with air for both the dry (air) and water-saturated cases. By imposing a temperature gradient, $\partial T / \partial x_{i}$ across the ends of the sample, and backing out the heat flux conducted, effective thermal conductivity may be readily estimated using Eq. (4). For these computations, the sidewalls are adiabatic and the calculation is performed in each coordinate direction for all the subvolumes.

The effective thermal conductivity was observed to be directionally dependent, as also noted for the case of metal foams in Bodla et al. [17]. Differences on the order of $15-20 \%$ were observed for the same subvolume along different coordinate directions. This anisotropy was attributed to the large difference in the number of particles along the different directions-for the samples under consideration, there were as few as 3-7 particles along the smallest dimension, while particles along the longer direction numbered approximately 15-35. Multiple realizations (subvolumes) were considered to mitigate some of the domain size artifacts and the values were reported as averages along with the observed standard deviation. The computations showed that in contrast to high-porosity metal foams (e.g., Ref. [17]), the effective thermal conductivity for sintered microstructures is a strong function of the microstructure, 
and not simply the porosity. With nearly same porosities, the effective thermal conductivity of the smallest particle size sample was observed to be 3-4 times that of the largest particle size sample [21]. The computed values of effective thermal conductivity were compared against Maxwell-Eucken models and the effective medium theory (EMT) models described in Sec. 2.1. The recommended bounding upper- and lower-limit Maxwell correlations highly over- and underpredict the effective thermal conductivity of sintered copper beds. This difference was attributed to the nature of the microstructure for which these models were developed. While the models considered spheres with large separations, the sintered samples under consideration were drastically different in structure. The EMT model [20], on the other hand, assumes a random dispersion of phases and yielded results within $50 \%$ of the computed values [21]. Figure 4(b) compares the computed values of effective thermal conductivity against those predicted by these models. However, the models cannot account for differences in thermal conductivity observed at constant porosity for the samples. Also shown in Fig. $4(c)$ are representative temperature contours for estimating thermal conductivity of a $250-355 \mu \mathrm{m}$ sample.

It has been reported previously in a number of studies (e.g., Ref. [25]) that effective thermal conductivity of sintered beds is significantly higher compared to that of simple packed beds and cold pressed beds. This difference is attributed to the interparticle neck regions in the microstructure which offer a low-resistance path for conduction. However, this critical neck region was seldom quantified. Employing their microtomography data, Bodla et al. [21] quantified this neck region for the three samples by defining a necking ratio, $N r$ :

$$
N r=\frac{A_{\text {neck }}}{V_{T}}, \quad A_{\text {neck }}=\frac{A_{s f, \text { segmented }}-A_{s f}}{2}
$$

The pre-sintered packed bed of particles has zero interparticle contact area (point contact), while particle-particle contact area increases as sintering proceeds. The parameter $\mathrm{Nr}$ characterizes the amount of sintering through the measurement of interparticle contact area per unit volume in the post-sintered sample, which governs the effective thermal conductivity. Figure 4(d) shows particles identified by color in a post-sintering sample. The surface area $A_{s f \text {,segmented }}$ may then be simply estimated as the cumulative surface area of all the particles. The effective thermal conductivity was found to increase as $\mathrm{Nr}$ increases and vice versa. At a fixed porosity, this dependence of effective thermal conductivity on $\mathrm{Nr}$ was observed to be linear, as shown in Fig. 4(a).

4.2.3 Permeability, Ergun's Coefficient, and Friction Factor. Hydrodynamic performance was estimated by imposing a constant velocity boundary condition coupled with a pressure outlet boundary condition on the opposite end of the sample in [21]. Furthermore, all the boundaries transverse to the main flow direction were made symmetric, and a no-slip boundary condition was employed at the solid-fluid interface. The computed values of pressure drop in the fully developed region were correlated to a standard Forchheimer equation, Eq. (2), for arriving at the permeability and Ergun's coefficient. The computations were performed for a variety of inlet velocities in the Reynolds number $\left(\operatorname{Re}_{K}\right)$ range of $0-10$. Water is considered as the interstitial fluid for both pressure drop and convective heat transfer computations.

Permeability was observed to be a direct function of particle/ pore size, i.e., the sample with the highest particle size exhibited the highest permeability and vice versa. The results were compared against the popular, semiempirical Carman-Kozeny correlation [1], developed for spherical particle beds. This correlation predicted permeability approximately three times the computed

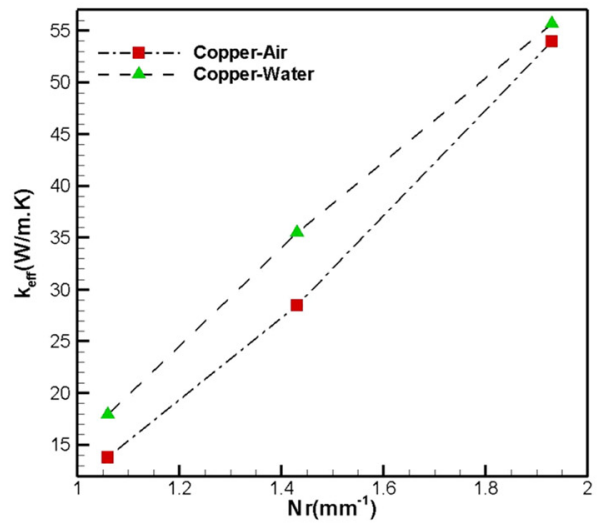

a)

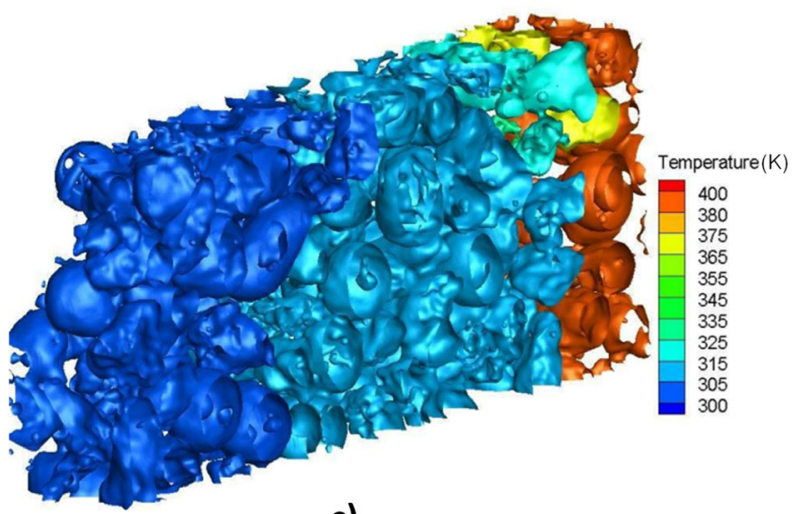

c)

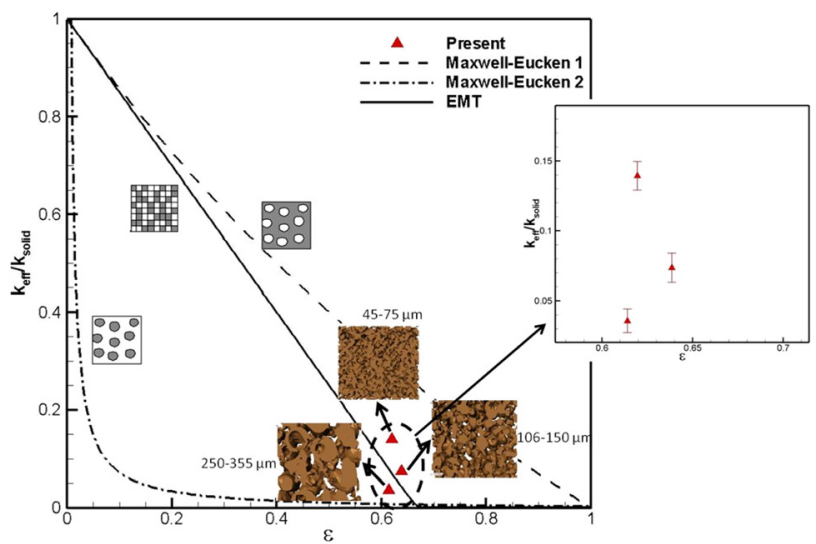

b)

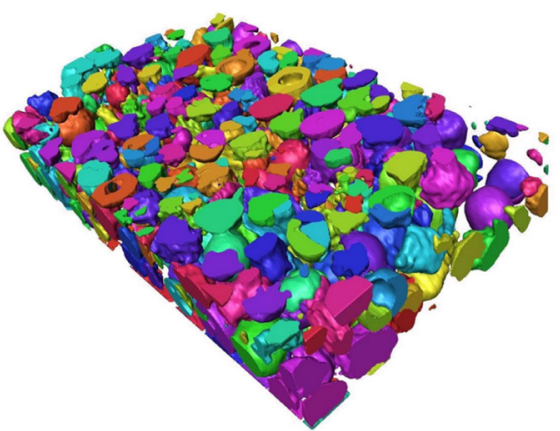

d)

Fig. 4 (a) Effective thermal conductivity as a function of necking ratio, and (b) comparison with commonly employed analytical models. Also shown are representative conduction contours in (c), along with particles identified by color in (d), for a $250-355 \mu \mathrm{m}$ sample. 
values. This difference was attributed to the nonspherical particle shape and the semiconsolidated nature of the porous sintered beds. In addition to permeability and Ergun's coefficient, the friction factor was also estimated and the results were compared against the correlation of Macdonald et al. [28] developed for smooth spherical particles. The correlation was observed to predict the computed values well in the viscous flow regime, where pressure drop is a linear function of velocity. However, at higher Reynolds numbers, the inertial component of pressure drop dominates, and the computed values were found to differ from the predicted values [21].

4.2.4 Interfacial Heat Transfer. The interfacial heat transfer coefficient and the corresponding Nusselt number were estimated for the case of thermally fully developed flow by imposing a constant heat flux boundary condition on the solid-fluid interface; for laminar flow, the results from this assumption would differ only by $10-15 \%$ relative to a constant temperature assumption. Heat transfer coefficient is calculated as:

$$
h=\frac{q^{\prime \prime}}{\bar{T}_{s}-T_{\text {in }}}
$$

Figure 5 shows streamtraces through a $250-355 \mu \mathrm{m}$ subvolume along with temperature contours. Also shown are computed values of Nusselt number compared against the Wakao and Kaguei correlation [29]. While this correlation developed for spherical particle beds agrees in trends with the computations, it does not provide accurate quantitative predictions of the interfacial heat transfer through sintered beds. In view of these limitations, new correlations, employing a similar functional relationship as in the Wakao and Kaguei correlation, were proposed based on computed values [21]. The correlations may be employed to predict interfacial heat transfer through random sintered beds of $\sim 60 \%$ porosity, and for Reynolds numbers in the Darcy regime $\left(\operatorname{Re}_{D_{p}} \approx 0-10\right)$.

4.3 Evaporation Characteristics. Bodla et al. [73] estimated two-phase characteristics of interest, viz. capillary pressure, effective pore radius, percentage thin film area, and evaporative mass and heat fluxes, for sintered-powder wick structures using the volume of fluid (VOF) model. Two different solution strategies were employed to stabilize the numerical solution and to improve convergence. After verifying these stabilization strategies, the VOF model was used to obtain static meniscus shapes in the pore space of the sintered wick samples. A steady-state evaporation analysis was performed using a modified Schrage equation [73] to model liquid-vapor phase change.
The evaporation analysis was performed for three sintered wick samples. The VOF method being a computationally expensive modeling technique, reduced domain sizes were needed as compared to the previously discussed finite volume approaches. Smaller volumes, with a typical size of approximately $0.5 \mathrm{~mm} \times 0.5 \mathrm{~mm}$ $\times 0.5 \mathrm{~mm}$, were considered for detailed, pore-scale evaporation analysis. Furthermore, only the pore space was considered under the assumption of an isothermal boundary condition for the solid wall. To lower the computational time without loss of accuracy, the as-produced mesh from SIMPLEWARE was further processed and coarsened using the commercial mesh generation package ICEM CFD [74], as discussed in Bodla et al. [73].

4.3.1 Volume of Fluid Method. Accurate numerical description of the interface motion and interfacial physics are a prelude to comprehensive numerical modeling of multiphase flows. Traditionally, the various numerical techniques used for describing the motion of the liquid-vapor interface have been categorized as interface tracking methods or interface capturing methods. In the former approaches, such as the Marker and Cell (MaC) method and arbitrary Lagranigian-Eulerian (ALE) method (e.g., Ref. [75]), the interface is tracked in a Lagrangian fashion. In the latter approach, the interface description is "captured" implicitly, and the interface motion is described in an Eulerian manner, i.e., by solving equations for an auxiliary interface function on a fixed grid. The VOF method, originally proposed by Hirt and Nichols [76], is a widely used interface-capturing method in which a color function, or volume fraction field, $\alpha(0 \leq \alpha \leq 1)$, is used to describe the interface separating the liquid and vapor phases. Beginning with an initialized volume fraction field, a continuity equation called the VOF equation, is solved for the secondary (heavier) phase. The volume fraction of the primary (lighter) fluid is thus obtained via volume conservation in each cell [76].

4.3.2 Numerical Modeling. In Ref. [73], the VOF model available in FLUENT [77] was employed for obtaining meniscus shapes in the complex pore domain of the scanned sintered wick microstructures. A piecewise linear interface reconstruction method was employed in conjunction with the continuum surface field (CSF) model of Brackbill et al. [78] for including the effect of surface tension in the momentum equations. Furthermore, to stabilize the meniscus oscillations and to reduce spurious currents due to the CSF model, a density ratio of unity was assumed for fluids on either side of the meniscus. However, the correct contact angle and surface tension, representative of the case of water vapor surrounding water, was imposed. For a static meniscus, as the flow field goes to zero at convergence, the choice of an unphysical density ratio will not affect the final solution so long as the Bond number is

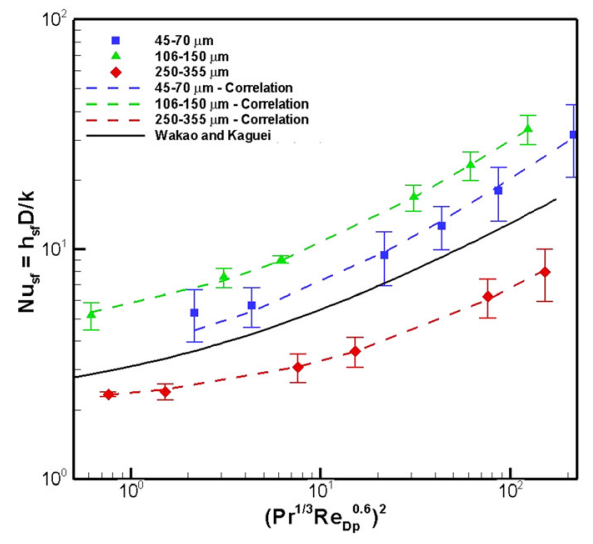

a)

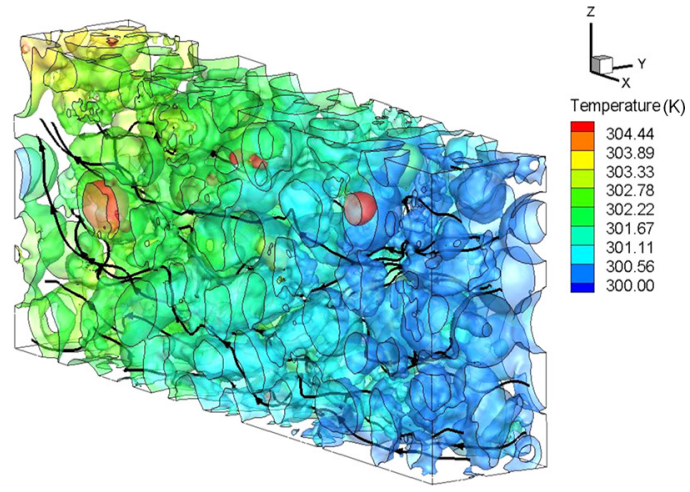

b)

Fig. 5 (a) Comparison of Nusselt number as a function of Reynolds number between microtomography-based computations and the correlation of Wakao and Kaguei [29], developed for spherical particle beds, and $(b)$ representative temperature contours and particle pathlines inside a $250-355 \mu \mathrm{m}$ sample 

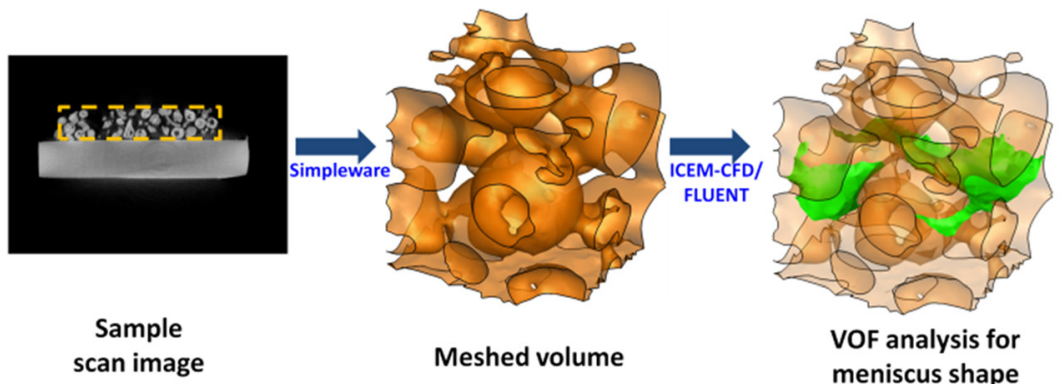

scan image

Meshed volume

VOF analysis for

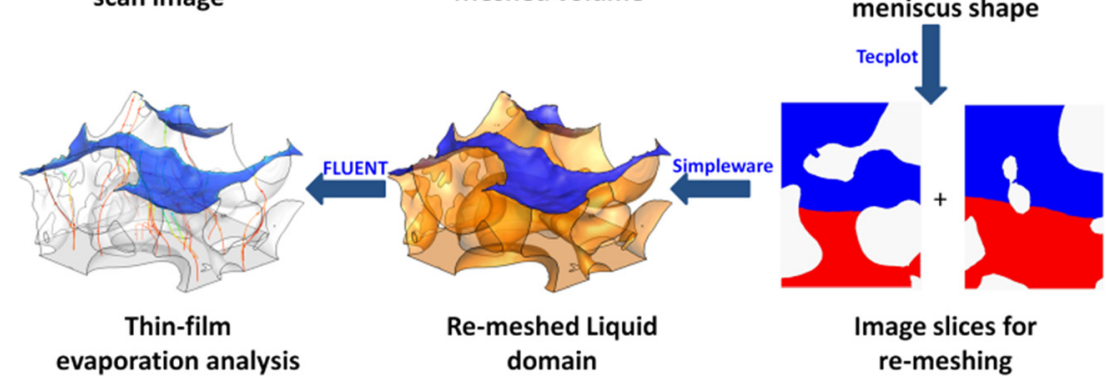

Fig. 6 Typical workflow employed in Ref. [73] for meshing, meniscus shape prediction and subsequent flow, heat transfer, and evaporation analyses

sufficiently low [73]; for the length scales of interest, the Bond number was $\mathrm{O}\left(10^{-3}\right)$. A second modification, increasing the viscosity of the fluid by two orders of magnitude, was also employed to promote viscous damping. The effects of these modifications were analyzed for the case of a two-dimensional meniscus formed in the interstitial space of two spherical particles. It was observed that these modifications only change the path to solution, but the final converged result remained the same [73]. Significant savings in computational time were realized.

Once the interface shape satisfying the prescribed boundary condition of the contact angle is obtained, an evaporation analysis was performed for the case of a static, continuously fed, evaporating meniscus. A low value of the Capillary number, which is $\mathrm{O}\left(10^{-3}-10^{-4}\right)$ for the low superheats considered, justifies the static meniscus assumption [73]. Following Ranjan et al. [55], flow and heat transport are solved in only the liquid domain. Moreover, the vapor was assumed to be saturated at constant pressure, consistent with steady operation of a heat pipe. Evaporation at the liquid-vapor interface was modeled via a modified Schrage expression based on kinetic theory [54,79], which was implemented in FLUENT with user defined functions [55]. The resulting mass flux was implemented as a corresponding volumetric source term in the near-interface cells and the corresponding energy transfer was modeled via an interfacial heat transfer coefficient. The simulations were performed for the case of a reference saturation temperature of $298 \mathrm{~K}$ and a superheat of $2 \mathrm{~K}$, following Ref. [55]. The specific modeling details and boundary conditions may be found in Bodla et al. [73]. The overall workflow, from 3D scanning to full evaporation analysis, is summarized in Fig. 6.

4.3.3 Capillary Pressure and Effective Pore Radius. The model was first verified and validated for the case of the meniscus in a 2D capillary pore formed between spheres, and then employed to estimate capillary pressure and effective pore radius of scanned sintered wick microstructures. Once the equilibrium shape was obtained for a prescribed contact angle, capillary pressure was estimated as the pressure difference across the meniscus [73]. The calculations were performed for a variety of contact angles in the range $30-75 \mathrm{deg}$, representative of typically observed contact angles in such media. It was observed that capillary pressure decreased as the contact angle increased for all the samples analyzed. Furthermore, at any contact angle, the smallest particle-size sample demonstrated the highest capillary pressure and vice versa. Based on the computations, capillary pressure was correlated to contact angle as a simple second-order curve fit. The effective pore radius was also calculated using a modified Young-Laplace equation, as follows:

$$
r_{\text {pore,eff }}=\frac{2 \sigma \cos \theta}{\Delta P_{\text {cap }}}
$$

This effective pore radius is unique to the microstructure geometry and accounts for the contact angle and surface tension. It may be used for estimating the capillary pressure that could be expected for a different working fluid or fluid-vapor combination.

4.3.4 Evaporative Heat Transfer Characteristics. The meniscus shapes obtained for the various samples are assumed to be static in analyzing the evaporative heat transfer performance. The 3D meniscus and the corresponding liquid domain were remeshed employing $2 \mathrm{D}$ slices obtained by post processing the solution data [73]. A number of characteristics, viz. evaporative heat transfer coefficient, evaporative mass and heat fluxes, overall wick resistance, and thinfilm area percentage, were estimated as summarized here.

All three samples exhibited a similar local evaporative heat transfer coefficient, averaged over the entire meniscus, establishing that the overall heat transfer may be improved by simply increasing the total meniscus surface area per unit volume. One such way is to reduce the particle/pore size. Figure 7(a) shows evaporative heat flux data as a function of contact angle for the three samples. For all the particle sizes, the evaporative mass flux and the corresponding heat flux were found to increase with decreasing contact angle, as a decrease in contact angle leads to an increase in overall meniscus surface area. Furthermore, as the contact angle increases, the portion of total evaporation occurring from the thin-film region increases. The thin-film area percentage of the total meniscus surface area was observed to be more or less independent of the contact angle, making it only a function of particle size-the percentage of thin-film area increased with decreasing particle size. Also shown in Fig. 7(b) are representative temperature contours and pathlines. The temperature contours indicate that similar results may be obtained by considering a conduction-only analysis, as also observed by Ranjan et al. [55].

\section{Reverse Engineering and Process-Based Simulation}

The final approach discussed in this article is the reverse engineering of porous media for desired fluid and thermal transport 


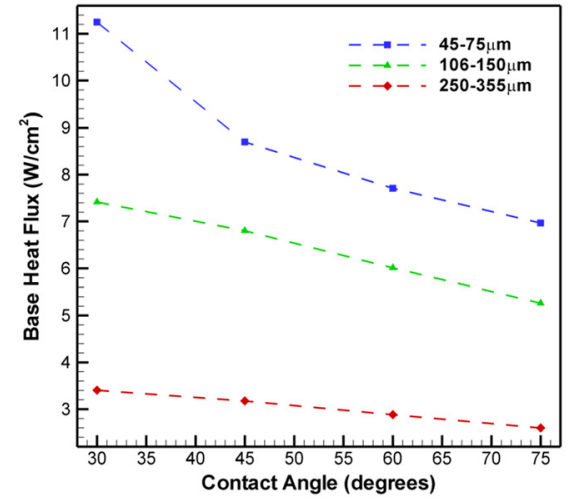

a)
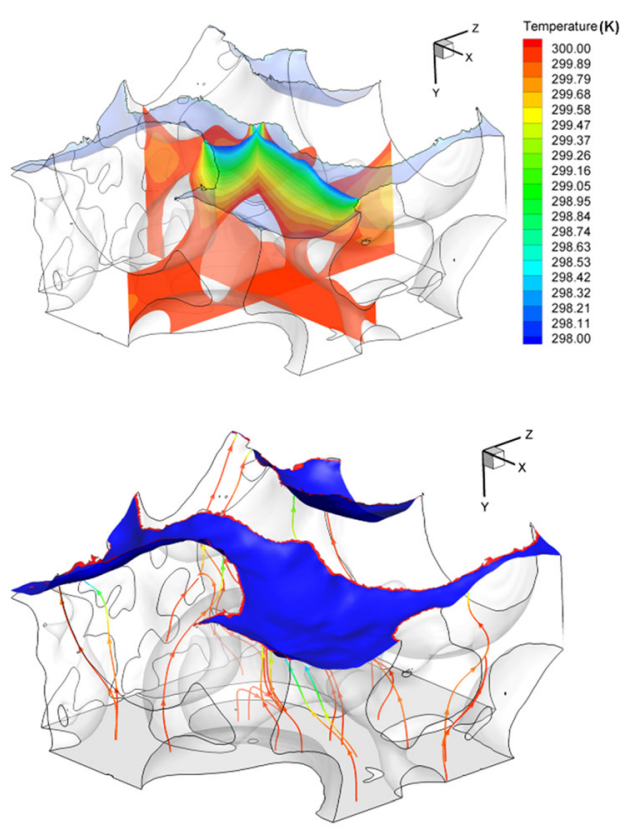

b)

Fig. 7 (a) Prediction of evaporative base heat flux as a function of contact angle, and (b) representative temperature contours and particle pathlines for evaporation colored by temperature for a $250-355 \mu \mathrm{m}$ particle size sintered powder sample

characteristics. Reverse engineering has been an active, steadily growing area of research pertaining to the optimization of porous materials, facilitated by advances in computing power and process-based simulation. The porous materials and their transport characteristics may be optimized from the initial stage of fabrication. The importance to thermal performance of a complete description and design of the microstructure morphology has been repeatedly demonstrated in the previous sections. For example, samples of different features, such as pore size for the case of foams (Ref. [80]) and particle size for the case of sintered beds (Sec. 4.2), exhibit vastly different thermal characteristics at fixed porosity. Similarly, keeping the feature size constant and varying the porosity yields significantly different performance. Processbased simulation approaches to microstructure generation from first principles allow an evaluation of the influence of the fabrication techniques on various geometric parameters, and hence on the ultimate performance. Such approaches have been developed in the past for cement-based materials [81,82], sedimentation in rocks [83], grain growth in metals [84], and ceramics [85]. For a thorough discussion of the various approaches in generating threedimensional digital microstructures, readers are referred to Garboczi et al. [86]. The discussion here is confined to porous sintered beds with relevance to enhancing thermal and fluid transport characteristics. Recent developments in process-based porous media modeling and potential manufacturing processes for fabricating prototype reverse-engineered porous media are discussed.

5.1 Microstructure Development During Sintering. Sintering is a material bonding phenomenon that occurs under the application of heat. Beginning with a loose powder compact, monolithic parts consisting of a collection of particles and interparticle connections (necks) are obtained by heating the material and holding it at a temperature below the melting point [87]. This bonding leads to a significant improvement in a number of structural and thermal characteristics of the part being sintered, such as improved strength, thermal conductivity and capillary pressure [10]. Sintering may proceed via surface diffusion, volume (lattice) diffusion, grain boundary diffusion, or evaporation-condensation diffusion mechanisms. German [87] provided a detailed description of these mechanisms; depending on characteristics such as particle size, temperature, and material properties (Eq. (8)), one or several of these could be the dominant mechanisms.

Sintering is often described to occur in three stages: an initial, intermediate, and final stage. The initial stage begins with a loose powder compact, wherein particles are in point contact. Interparticle connections begin to form as material diffuses to the neck region from the rest of the surface, and the pores begin to smoothen. In the intermediate stage, the pore space smoothens further, and the distinction between individual particles begins to fade. In this stage, the pores are still largely connected with each other [87]. In the final stage, the pore spaces become disconnected, and start to collapse if there are no interstitial trapped gases. Significant shrinkage and density change occurs and the individual grains (particles) are no longer distinguishable. Different diffusion mechanisms are dominant at these different stages; however, it must be noted that sintering is a continuous process and these stages overlap, with multiple dominant mechanisms active at any instant of time. Discrete stage distinctions have been made in literature for the purpose of describing the sintering physics and modeling the microstructure evolution [87].

Sintering theories describing the idealized case of two uniformly sized spheres have been well developed (e.g., Refs. $[88,89])$. Depending on the type of diffusion mechanism being modeled, different rates of neck growth are obtained. In general, for the various diffusion mechanisms, the growth of neck size, $x$, relative to the particle size, temperature, and time of sintering is described by the following functional form:

$$
x^{n}=\frac{C(T)}{D^{m-n}} t
$$

The exponents $m$ and $n$ and the factor $C(T)$ are assigned values depending on the specific diffusion mechanism [87]. Such theories have been widely used for describing material transport due to sintering in simple particle configurations in two and three dimensions. A few attempts have also been made to include different-sized spheres to capture the effect of particle size variation, such as by Ref. [90]. Some of the popular approaches used for modeling 
sintering are the discrete element method (DEM), phase field simulation, and Monte Carlo methods [90].

Commercial sintered wick structures are typically composed of a random collection of particles of different sizes particles. Including large variations in particle sizes for predicting microstructural evolution is infeasible with the aforementioned methods owing to the high computational costs involved in treating material transport in a macroscopic volume down to the scale of the individual grains. Mathematically tracking a surface under the influence of its own dynamics is notoriously difficult. For the case at hand, the surface normal velocity is proportional to the divergence of the gradient of the curvature (in the coordinates of the surface); a nonlinear fourth-order PDE with moving boundary conditions results. As noted in Ref. [91], such calculations have either employed approximations or numerical techniques, and are always limited to simple cases. To address this challenge, Pimienta et al. [91] developed a model for simulating sintering evolution due to surface mass transport, driven by curvature differences. By estimating curvature of the surface indirectly from images, and relocating pixels (mass) based on the measured curvature differences, realistic cases of random particle collections in two dimensions were successfully simulated, and the algorithm was later extended to three dimensions [92]. In their approach, analysis begins by replacing an atomically stepped surface with a continuous depiction, represented digitally by pixels. The size of a pixel is a small fraction of a particle, but far larger than atomic length scales. Several advantages of using digital images may be identified. Random particle shapes and sizes may be included. Physical quantities and transport characteristics may be readily computed based on images, either by employing the naturally discretized pixels as computational elements or by employing image-based meshing techniques (Sec. 4.1.2).

Bodla and Garimella [93] performed such sintering computations by considering a random collection of spheres having a userdefined particle size distribution. The model was first validated against theory and past experiments pertaining to idealized cases of sintering of equally sized cylinders and spheres, to verify that the sintering kinetics and physics are captured in a realistic way. Figure 8 (a) shows representative results of effective thermal conductivity

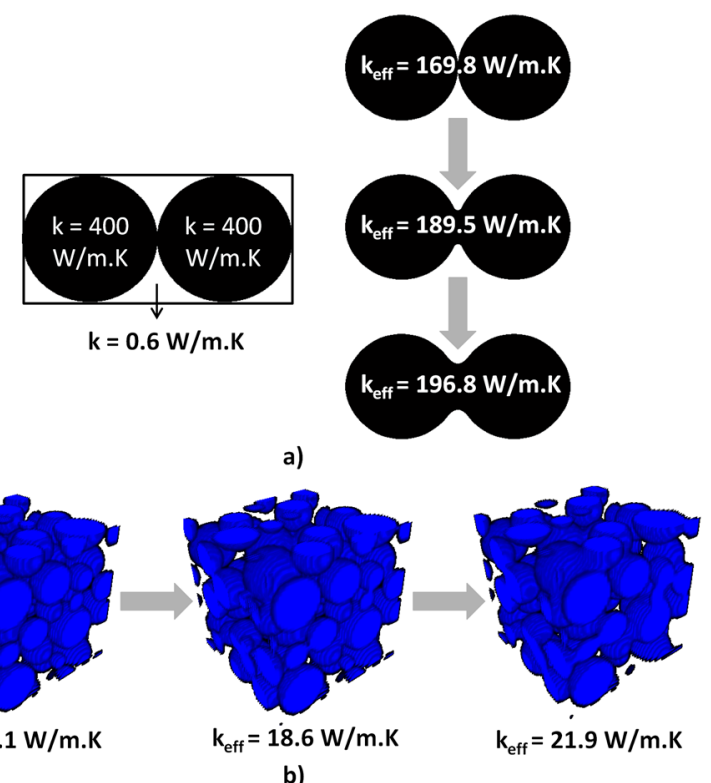

Fig. 8 (a) Two-particle sintering scenarios depicting sintering of cylindrical wires shown for a copper-water system, along with effective thermal conductivity calculated in the horizontal direction as a function of the extent of sintering, and (b) effective thermal conductivity of a random collection of spheres with near-Gaussian particle size distribution [93], as a function of extent of sintering and microstructure development as a function of amount of sintering for the case of two equally sized cylinders, a case widely analyzed in the literature. It may be observed that the necking or interparticle bonding, demonstrative of sintering, is realistically captured. The model was then employed to analyze the effect of sintering conditions, viz. temperature, time, particle size, and porosity, on properties such as effective thermal conductivity. Furthermore, Fig. 8(b) shows representative 3D microstructures as a function of the amount of sintering for the case of a near-Gaussian particle size distribution in the range $100-300 \mu \mathrm{m}$, at a porosity of $\sim 63 \%$ as found in commercial wick microstructures [93]. The simulated sintered microstructures appear qualitatively similar to the actual sintered samples reconstructed from XMT data, such as the ones shown in Fig. 5(b). Moreover, the computed effective thermal conductivity values were also observed to be similar to values from a previous study on direct simulation of scanned microstructures [21]. It is clear that the use of such a model provides the ability to control the sintering conditions and inputs to tailor and optimize porous material properties.

It is expected that future investigations will increasingly rely upon such process-based simulations for optimizing microstructures for specific applications, such as sintering models for heat pipe and vapor chamber wicks. As sintering proceeds, increased particle-particle bonding and closing of the pore space simultaneously enhance thermal conduction and increase fluid flow pressure drop. Optimized sintering conditions may be sought by maximizing an application-specific weighted function of pressure drop and thermal conduction resistance. The effects of nonspherical particles on transport characteristics may also be explored; one example being the pyramidal/conical microstructures previously shown to enhance thin-film evaporation characteristics [52]. In addition, rapid prototyping processes for manufacturing of reverseengineered, optimized porous microstructures may enable experimental evaluation of such numerically postulated structures that are not limited by conventional fabrication constraints. With advances in $3 \mathrm{D}$ printing of metals, and techniques such as selective laser sintering (SLS) and direct metal laser sintering (DMLS), it will be increasingly feasible to manufacture such digitally represented, intricate, user-defined microstructures.

\section{Conclusions}

With the rapid developments in portable, consumer and military electronics, there is an increasing need to remove large amounts and fluxes of heat from ever-diminishing spaces. Apart from exploring new thermal management solutions, existing cooling solutions also need to be optimized for enhanced thermal transport. Phase change-based thermal management designs are suitable candidate transport mechanisms for addressing high heat fluxes on the order of several hundred $\mathrm{W} / \mathrm{cm}^{2}$. One such solution that involves passive transport of the working fluid is a heat pipe or vapor chamber which incorporates a porous wick structure. Modeling and analysis methodologies employed for analyzing thermal transport through low-porosity porous sintered beds have been discussed, although the approaches are not restricted to this class of porous media. These models may be categorized in order of increasing complexity as semiempirical models, unit-cell based simulations, and direct simulation models for reconstructed 3D, random microstructures. The first approach relies on empirical parameters, while the third is a purely computational approach. The intricacies and limitations of these various approaches are reviewed in detail. In addition, recent advancements in simulating microstructure evolution and the related transport characteristics from first principles are presented. Such techniques are useful in evaluating the effect of various input parameters on the resulting physical characteristics. These models may be used for reverse engineering the microstructure of random porous media, thereby optimizing the transport characteristics of interest, and enabling manufacture of engineered porous materials tailored to specific needs. 


\section{Acknowledgment}

The authors acknowledge support for this work from industry members of the Cooling Technologies Research Center, an NSF Industry/University Cooperative Research Center at Purdue University.

\section{Nomenclature}

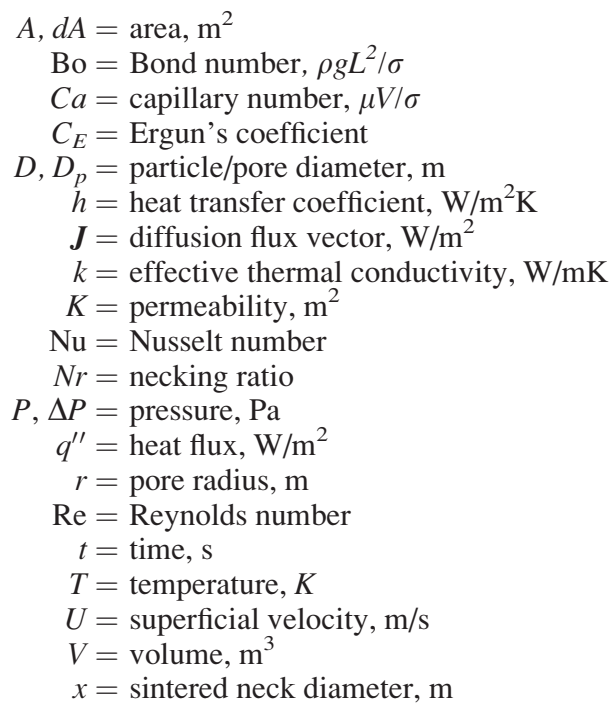

\section{Greek Symbols}

$$
\begin{aligned}
& \delta=\text { fitting parameter } \\
& \varepsilon=\text { porosity } \\
& \mu=\text { viscosity, } \mathrm{Ns} / \mathrm{m}^{2} \\
& \rho=\text { density, } \mathrm{kg} / \mathrm{m}^{3} \\
& \sigma=\text { surface tension, } \mathrm{N} / \mathrm{m} \\
& \theta=\text { contact angle, } \mathrm{rad}
\end{aligned}
$$

\section{Subscripts}

$$
\begin{aligned}
\text { eff } & =\text { effective } \\
f & =\text { fluid } \\
\text { in } & =\text { inlet } \\
p & =\text { pore/particle size } \\
s & =\text { solid } \\
s f & =\text { solid-fluid }
\end{aligned}
$$

\section{References}

[1] Kaviany, M., 2002, Principles of Heat Transfer, Wiley-Interscience, New York.

[2] Weibel, J. A., Garimella, S. V., and North, M. T., 2010, "Characterization of Evaporation and Boiling From Sintered Powder Wicks Fed by Capillary Action," Int. J. Heat Mass Transfer, 53, pp. 4204-4215.

[3] Bodla, K. K., Murthy, J. Y., and Garimella, S. V., 2012, "Optimization Under Uncertainty for Electronics Cooling Design Applications," Proceedings of the 13th IEEE Intersociety Conference on Thermal and Thermomechanical Phenomena in Electronic Systems, San Diego, CA, pp. 1191-1201.

[4] Garimella, S. V., and Sobhan, C. B., 2003, "Transport in Microchannels-A Critical Review," Ann. Rev. Heat Transfer, 13, pp. 1-50.

[5] Garimella, S. V., 2000, "Heat Transfer and Flow Fields in Confined Jet Impingement," Ann. Rev. Heat Transfer, 11, pp. 413-494.

[6] Garimella, S. V., Joshi, Y. K., Bar-Cohen, A., Mahajan, R., Toh, K. C., Carey, V. P., Baelmans, M., Lohan, J., Sammakia, B., and Andros, F., 2003, "Thermal Challenges in Next Generation Electronic Systems-Summary of Panel Presentations and Discussions," IEEE Trans. Compon. Packag. Technol., 25(4), pp. 569-575.

[7] Dewan, A., Mahanta, P., Raju, K. S., and Kumar, P. S., 2004, "Review of Passive Heat Transfer Augmentation Techniques," Proc. IMechE, Part A, 218(7), pp. $509-527$.

[8] Vafai, K., 2005, Handbook of Porous Media, CRC Press, Boca Raton, FL.

[9] Li, C., and Peterson, G. P., 2006, "Evaporation/Boiling in Thin Capillary Wicks (II)—Effects of Volumetric Porosity and Mesh Size," ASME J. Heat Transfer, 128(12), pp. 1320-1328.

[10] Faghri, A., 1995, Heat Pipe Science and Technology, Taylor \& Francis, Washington, DC.

[11] Peterson, G. P., 1994, An Introduction to Heat Pipes-Modeling, Testing, and Applications, John Wiley \& Sons, New York.
[12] Lin, Y.-Y., Semenic, T., and Catton, I., 2005, "Thermophysical Properties of Biporous Sintered Copper," Proceedings of the ASME Mechanical Engineering Congress and Exposition, Orlando, FL, pp. 785-791.

[13] Semenic, T., and Catton, I., 2009, "Experimental Study of Biporous Wicks for High Heat Flux Applications," Int. J. Heat Mass Transfer, 52(21), pp. 5113-5121.

[14] Weibel, J. A., and Garimella, S. V., 2012, "Visualization of Vapor Formation Regimes During Capillary-Fed Boiling in Sintered-Powder Heat Pipe Wicks," Int. J. Heat Mass Transfer, 55(13), pp. 3498-3510.

[15] Ingham, D. B., Bejan, A., Mamut, E., and Pop, I., 2003, Emerging Technologies and Techniques in Porous Media, Kluwer Academic Publishers, London.

[16] Vadász, P., 2008, Emerging Topics in Heat and Mass Transfer in Porous Media: From Bioengineering and Microelectronics to Nanotechnology, Springer, New York.

[17] Bodla, K. K., Murthy, J. Y., and Garimella, S. V., 2010, "MicrotomographyBased Simulation of Transport Through Open-Cell Metal Foams," Numer. Heat Transfer, Part A, 58(7), pp. 527-544.

[18] Krishnan, S., Murthy, J. Y., and Garimella, S. V., 2006, "Direct Simulation of Transport in Open-Cell Metal Foam," ASME J. Heat Transfer, 128(8), pp 793-799.

[19] Krishnan, S., Murthy, J. Y., and Garimella, S. V., 2005, "A Two-Temperature Model for Solid-Liquid Phase Change in Metal Foams," ASME J. Heat Transfer, 127(9), pp. 995-1004.

[20] Carson, J. K., Lovatt, S. J., Tanner, D. J., and Cleland, A. C., 2005, "Therma Conductivity Bounds for Isotropic Porous Materials," Int. J. Heat Mass Transfer, 48(11), pp. 2150-2158.

[21] Bodla, K. K., Murthy, J. Y., and Garimella, S. V., 2012, "Direct Simulation of Thermal Transport Through Sintered Wick Microstructures," ASME J. Heat Transfer, 134(1), p. 012602.

[22] Nozad, I., Carbonell, R. G., and Whitaker, S., 1985, "Heat Conduction in Multiphase Systems-I: Theory and Experiment for Two-Phase Systems," Chem. Eng. Sci., 40(5), pp. 843-855.

[23] Prasad, V., Kladias, N., Bandyopadhaya, A., and Tian, Q., 1989, "Evaluation of Correlations for Stagnant Thermal Conductivity of Liquid-Saturated Porous beds of Spheres," Int. J. Heat Mass Transfer, 32(9), pp. 1793-1796.

[24] Alexander, E. G., 1972, "Structure-Property Relationships in Heat Pipe Wicking Materials," Ph.D. thesis, North Carolina State University, Raleigh, NC.

[25] Atabaki, N., and Baliga, B. R., 2007, "Effective Thermal Conductivity of Water-Saturated Sintered Powder-Metal Plates," Int. J. Heat Mass Transfer, 44(1), pp. 85-99.

[26] Hadley, G. R., 1986, "Thermal Conductivity of Packed Metal Powders," Int J. Heat Mass Transfer, 29(6), pp. 909-920.

[27] Peterson, G. P., Fletcher, L. S., Raghunathan, S. R., Gillan, M. A., and Mitchell, R. D., 1987, "Effective Thermal Conductivity of Sintered Heat Pipe Wicks," J. Thermophys. Heat Transfer, 1(4), pp. 343-347.

[28] Macdonald, I. F., El-Sayed, M. S., Mow, K, and Dullien, F. A. L., 1979 "Flow Through Porous Media-The Ergun Equation Revisited," Ind. Eng. Chem. Fundam., 18(3), pp. 199-208.

[29] Wakao, N., and Kaguei, S., 1982, Heat and Mass Transfer in Packed Beds, Gordon and Breach Science, New York.

[30] Iverson, B. D., Davis, T. W., Garimella, S. V., North, M. T., and Kang, S. S., 2007, "Heat and Mass Transport in Heat Pipe Wick Structures," J. Thermophys. Heat Transfer, 21(2), pp. 392-404.

[31] Davis, T. W., and Garimella, S. V., 2008, "Thermal Resistance Measurement Across a Wick Structure Using a Novel Thermosyphon Test Chamber," Exp. Heat Transfer, 21(2), pp. 143-154.

[32] Migliaccio, C. P., and Garimella, S. V., 2011, "Evaporative Heat and Mass Transfer From the Free Surface of a Liquid Wicked Into a Bed of Spheres," Int J. Heat Mass Transfer, 54(15), pp. 3440-3447.

[33] Hanlon, M. A., and Ma, H. B., 2003, "Evaporation Heat Transfer in Sintered Porous Media," ASME J. Heat Transfer, 125(4), pp. 644-652.

[34] Adkins, D. R., and Dykhuizen, R. C., 1993, Procedures for Measuring the Properties of Heat-Pipe Wick Materials, Sandia National Labs, Albuquerque, NM.

[35] Imura, H., Kozai, H., and Ikeda, Y., 1994, "The Effective Pore Radius of Screen Wicks," Heat Transfer Eng., 15(4), pp. 24-32.

[36] Lin, Y.-Y., Semenic, T., and Catton, I., 2005, "Thermophysical Properties of Monoporous Sintered Copper," Proceedings of the ASME Summer Heat Transfer Conference, San Francisco, CA, pp. 17-23.

[37] Holley, B., and Faghri, A., 2006, "Permeability and Effective Pore Radius Measurements for Heat Pipe and Fuel Cell Applications," Appl. Therm. Eng. 26(4), pp. 448-462.

[38] Katz, A. J., and Thompson, A. H., 1985 "Fractal Sandstone Pores: Implications for Conductivity and Pore Formation," Phys. Rev. Lett., 54(12), pp. 1325-1328.

[39] Tyler, S. W., and Wheatcraft, S. W., 1989, "Application of Fractal Mathematics to Soil Water Retention Estimation," J. Soil Sci. Soc. Am., 53(4), pp. 987-996.

[40] Majumdar, A., 1992, "Role of Fractal Geometry in the Study of Thermal Phenomena," Ann. Rev. Heat Transfer, 4(4), pp. 51-110.

[41] Kitaoka, H., Takaki, R., and Suki, B., 1999, "A Three-Dimensional Model of the Human Airway Tree,” J. Appl. Physiol., 87(6), pp. 2207-2217.

[42] Yu, B., 2008, “Analysis of Flow in Fractal Porous Media," ASME Appl. Mech. Rev., 61(5), p. 050801.

[43] Ma, Y., Yu, B., Zhang, D., and Zou, M., 2003, "A Self-Similarity Model for Effective Thermal Conductivity of Porous Media," J. Phys. D: Appl. Phys. 36(17), pp. 2157-2164

[44] Ma, Y., Yu, B., Zhang, D., and Zou, M., 2004, "Fractal Geometry Model for Effective Thermal Conductivity of Three-Phase Porous Media," J. Appl. Phys. 95(11), pp. 6426-6434. 
[45] Yu, B., Lee, L. J., and Cao, H., 2002, "A Fractal In-Plane Permeability Model for Fabrics,” J. Polym. Compos., 23(2), pp. 201-221.

[46] Yu, B., and Cheng, P., 2002, "A Fractal Permeability Model for Bi-Dispersed Porous Media,” Int. J. Heat Mass Transfer, 45(14), pp. 2983-2993.

[47] Xu, P., and Yu, B., 2008, "Developing a New Form of Permeability and Kozeny-Carman Constant for Homogeneous Porous Media by Means of Fractal Geometry," Adv. Water Resour., 31(1), pp. 74-81.

[48] Vadakkan, U., Garimella, S. V., and Murthy, J. Y., 2004, "Transport in Flat Heat Pipes at High Heat Fluxes From Multiple Discrete Sources," ASME J. Heat Transfer, 126(3), pp. 347-354.

[49] Ranjan, R., Murthy, J. Y., Garimella, S. V., and Vadakkan, U., 2011, “A Numerical Model for Transport in Flat Heat Pipes Considering Wick Microstructure Effects," Int. J. Heat Mass Transfer, 54(1), pp. 153-168.

[50] Maxwell, J. C., 1873, A Treatise on Electricity and Magnetism, Clarendon Press, London.

[51] Domínguez Espinosa, F. A., 2011, "Effect of Fabrication Parameters on Thermophysical Properties of Sintered Wicks," M.S. thesis, Massachusetts Institute of Technology, Cambridge, MA

[52] Ranjan, R., Patel, A., Garimella, S. V., and Murthy, J. Y., 2011, "Wicking and Thermal Characteristics of Micropillared Structures for Use in Passive Heat Spreaders," Int. J. Heat Mass Transfer, 55, pp. 586-596.

[53] Ranjan, R., Murthy, J. Y., and Garimella, S. V., 2009, "Analysis of the Wicking and Thin-Film Evaporation Characteristics of Microstructures," ASME J. Heat Transfer, 131(10), p. 101001.

[54] Wang, H., Garimella, S. V., and Murthy, J. Y., 2007, "Characteristics of an Evaporating Thin Film in a Microchannel," Int. J. Heat Mass Transfer, 50(19-20), pp. 3933-3942.

[55] Ranjan, R., Murthy, J. Y., and Garimella, S. V., 2011, "A Microscale Model for Thin-Film Evaporation in Capillary Wick Structures," Int. J. Heat Mass Transfer, 54(1-3), pp. 169-179.

[56] Dhavaleswarapu, H. K., Garimella, S. V., and Murthy, J. Y., 2009, "Microscale Temperature Measurements Near the Triple Line of an Evaporating Thin Liquid Film," ASME J. Heat Transfer, 131(6), p. 061501.

[57] Migliaccio, C. P., Dhavaleswarapu, H. K., and Garimella, S. V., 2011, "Temperature Measurements Near the Contact Line of an Evaporating Meniscus V-Groove,” Int. J. Heat Mass Transfer, 54(7), pp. 1520-1526.

[58] Van Meerbeek, B., Vargas, M., Inoue, S., Yoshida, Y., Perdigao, J., Lambrechts, P., and Vanherle, G., 2000, "Microscopy Investigations: Techniques, Results, Limitations," Am. J. Dent., 13, pp. 3D-18D.

[59] Giannuzzi, L. A., and Stevie, F. A., 1999, "A Review of Focused Ion Beam Milling Techniques for TEM Specimen Preparation," Micron, 30(3), pp 197-204.

[60] Herman, G. T., 2009, Fundamentals of Computerized Tomography: Image Reconstruction From Projections, Springer, New York.

[61] Feldkamp, L. A., Davis, L. C., and Kress, J. W., 1984, "Practical Cone-Beam Algorithm," J. Opt. Soc. Am. A, 1(6), pp. 612-619.

[62] Stauber, M., and Muller, R., 2008, "Micro-Computed Tomography: A Method for the Non-Destructive Evaluation of the Three-Dimensional Structure of Biological Specimens," Methods Mol. Biol., 455, pp. 273-292.

[63] Jasti, J., Jesion, G., and Feldkamp, L., 1990, "Microscopic Imaging of Porous Media Using X-ray Computer Tomography," Proceedings of the SPE Annual Technical Conference and Exhibition, New Orleans, LA, pp. 27-34.

[64] Siebert, J. E., Petrovic, A. M., and Rieke, P. E., 1982, "Soil Bulk Density Analysis in Three Dimensions by Computed Tomographic Scanning," Soil Sci. Soc. Am. J., 46(3), pp. 445-450.

[65] Anderson, S. H., Peyton, R. L., and Gantzer, C. J., 1990, "Evaluation of Constructed and Natural Soil Macropores Using X-ray Computed Tomography," Geoderma, 46(1-3), pp. 13-29.

[66] Givler, R. C., and Altobelli, S. A., 1994, "A Determination of the Effective Viscosity for the Brinkman-Forchheimer Flow Model,” J. Fluid Mech., 258, pp $355-370$.

[67] Gleeson, J., Woessner, D., and Jordan, C., Jr., 1993, "NMR Imaging of Pore Structures in Limestones," SPE Formation Eval., 8(2), pp. 123-127.

[68] Young, P. G., Beresford-West, T. B., Coward, S. R., Notarberardino, B., Walker, B., and Abdul-Aziz, A., 2008, "An Efficient Approach to Converting
Three-Dimensional Image Data into Highly Accurate Computational Models," Philos. Trans. R. Soc. London, Ser. A, 366(1878), pp. 3155-3173.

[69] Kenesei, P., Borbely, A., and Biermann, H., 2004, "Microstructure Based Three-Dimensional Finite Element Modeling of Particulate Reinforced Metal-Matrix Composites," Mater. Sci. Eng. A, 387-389, pp. $852-856$.

[70] Youssef, S., Maire, E., and Gaertner, R., 2005, "Finite Element Modelling of the Actual Structure of Cellular Materials Determined by X-ray Tomography," Acta Mater., 53(3), pp. 719-730.

[71] Watson, I. G., Lee, P. D., Dashwood, R. J., and Young, P., 2006, "Simulation of the Mechanical Properties of an Aluminum Matrix Composite Using X-ray Microtomography," Metall. Mater. Trans. A, 37A(3), pp. 551-558.

[72] Simpleware Ltd., 2002, ScanIP, ScanFE and ScanCAD Tutorial Guide for SIMPLEWARE 3.1, 2002, Simpleware Ltd., Exeter, UK.

[73] Bodla, K. K., Murthy, J. Y., and Garimella, S. V., "Evaporation Analysis in Sintered Wick Microstructures," Int. J. Heat Mass Transfer (to be published).

[74] ANSYs, Inc., 2007, User's Manual for ANSYS ICEM CFD 11, ANSYs Inc., Canonsburg, PA.

[75] Souli, M., and Zolesio, J. P., 2001, “Arbitrary Lagrangian-Eulerian and Free Surface Methods in Fluid Mechanics," Comput. Methods Appl. Mech. Eng., 191(3-5), pp. 451-466.

[76] Hirt, C. W., and Nichols, B. D., 1981, "Volume of Fluid (VOF) Method for the Dynamics of Free Boundaries," J. Comput. Phys., 39(1), pp. 201-225.

[77] Fluent, Inc., 2002, User's Guide for FLuENT 6.0, Fluent Inc., Lebanon, NH.

[78] Brackbill, J. U., Kothe, D. B., and Zemach, C., 1992, "A Continuum Method for Modeling Surface Tension,” J. Comput. Phys., 100(2), pp. 335-354.

[79] Schrage, R. W., 1953, A Theoretical Study of Interface Mass Transfer, Columbia University Press, New York.

[80] Bodla, K. K., Murthy, J. Y., and Garimella, S. V., 2010, "Resistance NetworkBased Thermal Conductivity Model for Metal Foams," Comput. Mater. Sci. 50(2), pp. 622-632.

[81] Garboczi, E. J., and Bentz, D. P., 1991, "Fundamental Computer Simulation Models for Cement-Based Materials," Mater. Sci. Concr. II, Am. Ceram. Soc., 3, pp. 249-277.

[82] Garboczi, E. J., and Bentz, D. P., 1992, "Computer Simulation of the Diffusivity of Cement-Based Materials,” J. Mater. Sci., 27(8), pp. 2083-2092.

[83] Visscher, P. B., and Cates, J. E., 1990, "Simulation of Porosity Reduction in Random Structures," J. Mater. Res., 5(10), pp. 2184-2196.

[84] Srolovitz, D. J., Anderson, M. P., Sahni, P. S., and Grest, G. S., 1984 "Computer Simulation of Grain Growth-II. Grain Size Distribution, Topology, and Local Dynamics," Acta Metall., 32(5), pp. 793-802.

[85] Ku, W., Gregory, O. J., and Jennings, H. M., 1990, "Computer Simulation of the Microstructure Developed in Reaction-Sintered Silicon Nitride Ceramics," J. Am. Ceram. Soc., 73(2), pp. 286-296.

[86] Garboczi, E. J., Bentz, D. P., and Martys, N. S., 1999, "Digital Images and Computer Modeling," Experimental Methods for Porous Media, Academic Press, New York, pp. 1-41.

[87] German, R. M., 1996, Sintering Theory and Practice, John Wiley \& Sons, New York.

[88] Wakai, F., 2006, "Modeling and Simulation of Elementary Processes in Ideal Sintering," J. Am. Ceram. Soc., 89(5), pp. 1471-1484.

[89] Wakai, F., and Aldinger, F., 2003, "Sintering Through Surface Motion by the Difference in Mean Curvature," Acta Mater., 51(14), pp. 4013-4024.

[90] Kumar, V., 2011, "Simulations and Modeling of Unequal Sized Particles Sintering," Ph.D. thesis, University of Utah, Salt Lake City, UT.

[91] Pimienta, P. J. P., Garboczi, E. J., and Carter, W. C., 1992, "Cellular Automaton Algorithm for Surface Mass Transport Due to Curvature Gradients: Simulations of Sintering," Comput. Mater. Sci., 1(1), pp. 63-77.

[92] Bentz, D. P., Pimienta, P. J. P., Garboczi, E. J., and Carter, W. C., 1991, "Cellular Automaton Simulations of Surface Mass Transport Due to Curvature Gradients: Simulations of Sintering in 3-Dimensions," Synth. Process. Ceram. 249, pp. 413-418.

[93] Bodla, K. K., and Garimella, S. V., "Microstructural Evoluation and Transport Properties of Sintered Porous Media," Proceedings of the Summer Heat Transfer Conference, Minneapolis, MN (to be published). 